\title{
Analysis of series with stochastic coefficients
}

\author{
R. Dekeyser \\ Instituut voor Theoretische Fysica, Katholieke Universiteit Leuven, B-3030 Leuven, Belgium \\ F. Iglói* \\ Institut für Theoretische Physik, Universität zu Köln, D-5000 Köln, Federal Republic of Germany \\ and Instituut voor Theoretische Fysica, Katholieke Universiteit Leuven, B-3030 Leuven, Belgium \\ F. Mallezie \\ Institurt voor Theoretische Fysica, Katholieke Universiteit Leuven, B-3030 Leuven, Belgium \\ F. Seno \\ Dipartimento di Fisica, Universitá di Padova, I-35100 Padova, Italy \\ and Instituut voor Theoretische Fysica, Katholieke Universiteit Leuven, B-3030 Leuven, Belgium
}

(Received 24 January 1989; revised manuscript received 29 March 1990)

\begin{abstract}
For functions with power-law singularities we consider series expansions whose coefficients have been determined by Monte Carlo simulation. In many practical problems the relative noise of the coefficients is constant or slowly increasing with the order. We modeled real Monte Carlo expansions by test series with known singularity structure where noise with different strength and form is imposed on the coefficients. The efficiency of different standard methods of series analysis (ratio method, Padé approximants, differental approximants) has been tested together with smoothing methods based on repeated partial summation of the series. We found the Padé method to give reasonable estimates and its accuracy is independent of the smoothing, while the estimates of the ratio and the differential approximant methods are greatly improved when smoothing is applied. Indeed, we found the ratio method with optimally selected smoothing to give the most reliable results.
\end{abstract}

\section{INTRODUCTION}

Different exact series-expansion methods ${ }^{1}$ combined with methods of series analysis ${ }^{2,3}$ are useful approaches to critical phenomena. The higher-order terms of an expansion, however, contain more and more configurational information such that one has to use sophisticated methods and complicated computer programs to calculate them. Therefore the number of coefficients is strongly limited by the available computer time.

In some geometrical critical problems (walks, ${ }^{4}$ percolation, ${ }^{5}$ lattice animals, ${ }^{6}$ etc.), however, it is natural to determine the coefficients of the expansion approximately by Monte Carlo (MC) simulation. ${ }^{7}$ In this waywith simpler computer programs and moderate computing effort-longer series can be calculated. For problems where the asymptotic region can be reached only in large order (cf. $k$-tolerant walks, ${ }^{4}$ i.e., walks that can visit each lattice site at most $k$ times) this is a necessary approach. Quite generally the values of any singular quantity determined by $\mathrm{MC}$ simulations on different finite systems form such a stochastic series. ${ }^{7}$

Having determined the series, the next problem is to deduce the critical exponent. So far mainly simple procedures based on the ratio method and combined with graphical interpretations have been used, but the more sophisticated methods developed to treat the confluent singularities of exact series are rarely applied. The reason for this is that for MC series the noise seems to play the principal role, and special procedures are necessary to reduce its effect. Presumably a smoothing based on repeated partial summation of the series ${ }^{8}$ is adequate for such a purpose. One hopes that after this transformation the fluctuations in the individual coefficients will be washed out such that the effect of noise on the estimate will be reduced.

In this paper we are interested in the role of noise on the estimates of different methods of series analysis and try to find out strategies to minimize the effect of stochasticity. For this purpose we mainly analyze test functions with given singularity structure where on the coefficients some noise with different strength and form is imposed. A typical form of the noise is deduced from scaling arguments and from examples of MC simulation.

The structure of the paper is the following. In Sec. II standard methods of series analysis (ratio method, Padé approximants, differential approximants) are shortly reviewed and a smoothing transformation based on repeated partial summation of the series is described. Different noisy series are analyzed in Sec. III, while the results are discussed in Sec. IV. 


\section{METHODS OF ANALYSIS AND SMOOTHING}

\section{A. Standard methods of series analysis}

Let us consider a series of the following elements:

$$
a_{1}, a_{2}, a_{3}, \ldots, a_{n}, \ldots
$$

which for large $n$ asymptotically behaves as

$$
a_{n}=A n^{\alpha}\left(1+b n^{-\beta}+c n^{-1}+\cdots\right)
$$

where $\alpha>0,0<\beta<1$. In critical phenomena there are many examples for such series, e.g., walks, lattice animals, percolation, or the finite-size behavior of singular quantities at the critical point. ${ }^{9}$ On the other hand, the power series with coefficients $a_{n}$

$$
f(x)=\sum_{n} a_{n} x^{n}
$$

defines a function, which has a power-law singularity at $x_{c}=1:^{8}$

$$
f(x) \sim(1-x)^{-(\alpha+1)} \text { for } x \rightarrow 1 .
$$

Various methods of analysis have been developed to determine the critical exponent $\alpha$ from a finite series. ${ }^{2,3}$

In the ratio method an estimate for $\alpha$ is obtained from the ratio of two subsequent coefficients $r_{n}=a_{n} / a_{n-1}$ as

$$
\alpha_{n}=n\left(r_{n}-1\right)
$$

On the other hand, in the differential approximant (DA) and Padé method each estimate depends on all coefficients. In the DA method $f(x)$ is the solution of the differential equation: ${ }^{10}$

$$
Q_{M}(x) f^{\prime}(x)+P_{L}(x) f(x)+R_{N}(x)=O\left(x^{M+L+N+2}\right)
$$

with $Q_{M}(0)=1$. Here the functions $Q_{M}(x), P_{L}(x)$, and $R_{N}(x)$ are polynomials of the order of $M, L$, and $N$, respectively, chosen in such a way that $\mathrm{Eq} .(2.6)$ is fulfilled up to $O\left(x^{M+L+N+2}\right)$. Then the solution $f(x)$ is called the $[N / L ; M]$ approximant.

Equation (2.6) contains as a special case the Padé method for $Q_{M}(x)=0$, and the solution is denoted as the $[N / L]$ approximant. Finally the $D \log$ Padé method corresponds to $R_{N}(x)=0$, with solutions denoted as the $[L / M]$ approximant.

\section{B. Smoothing by partial summation} $(2.1)$

Let us perform the following transformation on series

$$
a_{n}^{s}=\sum_{i=1}^{n} C_{n, i}^{s} a_{i}
$$

where the coefficients are expressed by the $\Gamma$ function:

$$
C_{n, i}^{s}=\frac{\Gamma(n-i+s)}{\Gamma(s) \Gamma(n-i+1)} .
$$

For $s=1$, obviously $C_{n, i}^{1}=1$; thus $\left\{a_{n}^{1}\right\}$ is the partially summed series of $\left\{a_{n}\right\} .^{8}$ Similarly for integer $s$ the $\left\{a_{n}^{s}\right\}$ give the $s$-times partially summed series.

Performing the summation in (2.7) one can see that for the transformed series both the leading and the nextto-leading exponents are shifted by $s$, but their relative strength has changed as

$$
a_{n}^{s}=A_{s} n^{\alpha+s}\left(1+b_{s} n^{-\beta}+c_{s} n^{-1}+\cdots\right)
$$

where the coefficients are

$$
\begin{aligned}
A_{s} & =A \frac{\Gamma(1+\alpha)}{\Gamma(s+1+\alpha)} \\
b_{s} & =b \frac{\Gamma(s+1+\alpha)}{\Gamma(1+\alpha)} \frac{\Gamma(1+\alpha-\beta)}{\Gamma(s+1+\alpha-\beta)}, \\
c_{s} & =(\alpha+s)\left(\frac{s}{2}+\frac{c}{\alpha}\right) .
\end{aligned}
$$

As one can see from Eq. (2.10) the strength of the confluent singularity monotonically increases with $s$. Since for noisy series appropriate smoothing is expected for positive $s$, one has to make a compromise, selecting $s$ optimally.

\section{ANALYSIS OF STOCHASTIC SERIES}

\section{A. The form of the noise}

Before starting to analyze $\mathrm{MC}$ series first we have to discuss their main properties: what is the typical form and strength of the noise in various coefficients? As an example we study the results of a MC simulation on the square of the end-to-end distance $R_{n}^{2}$ of a random walk of $n$ steps on a square lattice. The relative noise $\sigma$, i.e., the standard deviation divided by the average is given in Table I for 100 groups of $\mathrm{W}=1000$ walks. As is seen $\sigma(n)$ is fairly constant close to its asymptotic value $\sigma$ $\sim W^{-1 / 2}$.

This kind of behavior of the noise is typical for general self-avoiding walks, too. To show this we recall the probability function in the scaling regime ${ }^{11}$

TABLE I. Relative noise of $R_{n}^{2}$ for random walks in a MC simulation on the square lattice.

\begin{tabular}{rl}
\hline $\mathrm{N}$ & $\sigma(n)$ \\
\hline 5 & 0.0296 \\
10 & 0.0275 \\
20 & 0.0287 \\
30 & 0.0280 \\
40 & 0.0303 \\
50 & 0.0322 \\
60 & 0.0324 \\
70 & 0.0322 \\
80 & 0.0323 \\
90 & 0.0324 \\
100 & 0.0323 \\
\hline \hline
\end{tabular}




$$
P(R, n)=R^{-d} f\left(\frac{R}{n^{\nu}}\right),
$$

which measures the probability to find a walk with a distance $R$ from the starting point after $n$ steps. Using expression (3.1) it is easy to verify that the relative noise is indeed independent of $n$.

Based on these observations we believe that for a wide class of MC series the coefficients can be represented as

$$
a_{n}=a_{n}^{0}\left(1+\sigma \delta_{n}\right), \quad n=1,2, \ldots, N
$$

where $a_{n}^{0}$ denotes the exact value and $\delta_{n}$ is a random number with normal distribution, zero average, and standard deviation 1 .

In practice, if we want to determine the coefficients of a series with a given accuracy, the computer time necessary to calculate them rapidly increases with the order. Therefore in many algorithms the higher-order coefficients are determined with less accuracy than the lower-order ones. One possibility to model this behavior is to choose the relative noise as increasing linearly with the order:

$$
a_{n}=a_{n}^{0}\left(1+\sigma \frac{2 n}{N} \delta_{n}\right), \quad n=1,2, \ldots, N .
$$

Note that the average of the relative noise in this case is the same as for (3.2).

To test the different methods of analysis we use test functions with given singularity structure and with noise according to (3.2) and (3.3). The test functions listed in Table II are the simple power $(A)$, and functions $C, G$, and $K$, as given by Hunter and Baker. ${ }^{3}$ In practice we generated 100 series in each case and determined the average and the standard deviation of the estimates for the critical exponent. For $\sigma$ we used three values: 0.01, 0.05, and 0.2 , corresponding to an accurate $\mathrm{MC}$ simulation, to an average one, and to a poor one, respectively.

\section{B. Analysis of series without smoothing}

In the ratio method according to (2.5) the standard deviation of an estimate increases - at least linearly - with the order, since the uncertainty on $r_{n}$ is not a decreasing function of $n$. Consequently the ratio method without a further transformation is not applicable to analyze a noisy series.

TABLE II. Test functions used to study the effectiveness of different methods of series analysis. On the coefficients of their power-series noise of the form of Eqs. (3.2) and (3.3) is imposed.

\begin{tabular}{ll}
\hline \hline $\mathrm{A}$ & $(1-x)^{-1.5}$ \\
$\mathrm{C}$ & $(1-x)^{-1.5}\left(1-\frac{1}{2} x\right)^{1.5}+\mathrm{e}^{-x}$ \\
$\mathrm{G}$ & $(1-x)^{-1.5}+\left\{2(1-x)(2-x)^{6} /\left[(2-x)^{7}-x^{7}\right]\right\}^{1.25}$ \\
$\mathrm{~K}$ & $(1-x)^{-1.5}+\left(1+\frac{4}{5} x\right)^{-1.25}+\mathrm{e}^{-x}$ \\
\hline \hline
\end{tabular}

The Padé method, however, gives useful estimates without smoothing, too. In Table III the estimates and the standard deviations are listed for $N=20$ and 50 for constant and linear noise. In the calculation 16 central elements of the Padé table were taken, omitting, however, those terms which differ from the average more than the triple of the standard deviation. Then, as already described, the results are averaged over 100 series.

As one can see from Table III the estimates are reasonably good for smaller constant noise $(\sigma=0.01$ and 0.05 ), and the accuracy increases with $N$. For $\sigma=0.2$, however, the estimates scatter very much. Considering now the series with linear noise, we can say that the accuracy in this case is roughly the same as that with average constant noise. At first thought this result seems to be surprising, since for exact series the estimates will be greatly improved if higher-order terms are present in the analysis. On this basis one would expect less accuracy if the higher-order coefficients have larger fluctuations. In reality, however, the gain in accuracy for lower-order coefficients seems to compensate this effect.

Next, using the method of differential approximants we have performed the same analysis as for the Padé method, using the first-order inhomogeneous DA. (For the DA method 20 central elements of the table were taken together with the same selection procedure as for the Padé method.) According to the results in Table IV the estimates are reasonable for this method, although the accuracy is significantly less than for the Padé method. The form of the noise seems to have little effect on the estimates, in this case, too.

\section{The effect of smoothing}

The test series smoothed by the (3.1) transformation with $s=1,3,5$ are analyzed by the Padé and the DA methods. For these methods we found the difference between the exact exponents and the average of the estimates to be always smaller than the standard deviation of the estimates; therefore the effect of smoothing is conveniently characterized by the standard deviations which are given in Table $\mathrm{V}$ for constant noise with $\sigma=0.05$.

As is seen in Table $\mathrm{V}$ the Padé method is insensitive to the application of smoothing for all cases. As an explanation one could argue that the Padé method using the whole series for each estimate is insensitive to fluctuations in individual coefficients. This explanation, however, does not hold for the DA method, which works using the same principle. As one sees from Table $V$ the accuracy does depend on smoothing for the DA method, and at $s \sim 3$ this is comparable with that of the Padé method.

We now turn to discuss the effect of smoothing on the results of the ratio method, where-as we have already noted-it is necessary to perform some transformation before analysis. By the ratio method, due to its simplicity, one can easily determine a whole series of estimates $\alpha_{n}$ and more conveniently represent them graphically. In 
TABLE III. Analysis of test series with lengths $N=20$ and 50 for constant and linear noise with strengths $\sigma=0.01,0.05$ and 0.2 by the Dlog Padé method. $\Delta \alpha$ is the standard deviation on the obtained $\alpha$ values.

\begin{tabular}{|c|c|c|c|c|c|c|}
\hline \multirow[b]{2}{*}{ Function } & \multirow[b]{2}{*}{$\sigma$} & \multirow[b]{2}{*}{$N$} & \multicolumn{2}{|c|}{ Constant noise } & \multicolumn{2}{|c|}{ Linear noise } \\
\hline & & & $\alpha$ & $\Delta \alpha$ & $\alpha$ & $\Delta \alpha$ \\
\hline \multirow[t]{6}{*}{ A } & 0.01 & 20 & 1.503 & 0.021 & 1.501 & 0.019 \\
\hline & 0.05 & 20 & 1.509 & 0.095 & 1.511 & 0.095 \\
\hline & 0.20 & 20 & 1.564 & 0.324 & 1.469 & 0.487 \\
\hline & 0.01 & 50 & 1.500 & 0.014 & 1.501 & 0.008 \\
\hline & 0.05 & 50 & 1.505 & 0.046 & 1.526 & 0.138 \\
\hline & 0.20 & 50 & 1.507 & 0.302 & 1.510 & 0.260 \\
\hline \multirow[t]{6}{*}{$\mathrm{C}$} & 0.01 & 20 & 1.659 & 0.098 & 1.662 & 0.111 \\
\hline & 0.05 & 20 & 1.668 & 0.247 & 1.649 & 0.274 \\
\hline & 0.20 & 20 & 1.624 & 0.737 & 1.628 & 0.868 \\
\hline & 0.01 & 50 & 1.537 & 0.057 & 1.543 & 0.092 \\
\hline & 0.05 & 50 & 1.549 & 0.061 & 1.595 & 0.181 \\
\hline & 0.20 & 50 & 1.531 & 0.256 & 1.586 & 0.206 \\
\hline \multirow[t]{6}{*}{$\mathrm{G}$} & 0.01 & 20 & 1.409 & 0.058 & 1.419 & 0.058 \\
\hline & 0.05 & 20 & 1.505 & 0.095 & 1.497 & 0.285 \\
\hline & 0.20 & 20 & 1.555 & 0.350 & 1.632 & 0.302 \\
\hline & 0.01 & 50 & 1.502 & 0.017 & 1.497 & 0.018 \\
\hline & 0.05 & 50 & 1.499 & 0.069 & 1.495 & 0.050 \\
\hline & 0.20 & 50 & 1.485 & 0.187 & 1.514 & 0.163 \\
\hline \multirow[t]{6}{*}{$\mathrm{K}$} & 0.01 & 20 & 1.589 & 0.105 & 1.589 & 0.191 \\
\hline & 0.05 & 20 & 1.596 & 0.128 & 1.625 & 0.201 \\
\hline & 0.20 & 20 & 1.776 & 0.895 & 1.669 & 0.391 \\
\hline & 0.01 & 50 & 1.518 & 0.062 & 1.510 & 0.031 \\
\hline & 0.05 & 50 & 1.529 & 0.073 & 1.532 & 0.062 \\
\hline & 0.20 & 50 & 1.535 & 0.204 & 1.542 & 0.127 \\
\hline
\end{tabular}

the following we analyze the test series up to 50 terms using a constant noise of $\sigma=0.05$.

Let us start with function $A$ which represents the simplest case. The average of the estimates and its standard deviation for different values of $s$ are drawn in Fig. 1 as a function of $1 / n$. As one can see the standard deviation is decreasing with the order, and the optimal value of $s$ is about $s \sim 3$, where $\Delta \alpha$ is minimal. Due to the fact that function $A$ contains no confluent singularity there is no systematic $n$ dependence of $\alpha_{n}$. Therefore the accuracy of the estimates is high $\Delta \alpha<0.01$; it is much better than with any other method.

The strength of the confluent singularity seems to be small for function $G$, too, as one can see in Figs. 2 and 3 for $\sigma=0.05$ and 0.2 , respectively. The estimate is surprisingly accurate, even for strong noise $\sigma=0.2$, the standard deviation being less than $4 \%$.

The confluent singularity, however, plays an important role for functions $C$ and $K$, as one can see from Figs. 4 and 5. Although the standard deviations are relatively small $(\Delta \alpha \sim 0.015)$ in these cases, too, the estimates $\alpha_{n}$ contain systematic $1 / n$ corrections, the strength of which increases with the smoothing. ${ }^{12}$ Taking into account a $1 / n$ correction term the estimates are $\alpha=1.51 \pm 0.05$

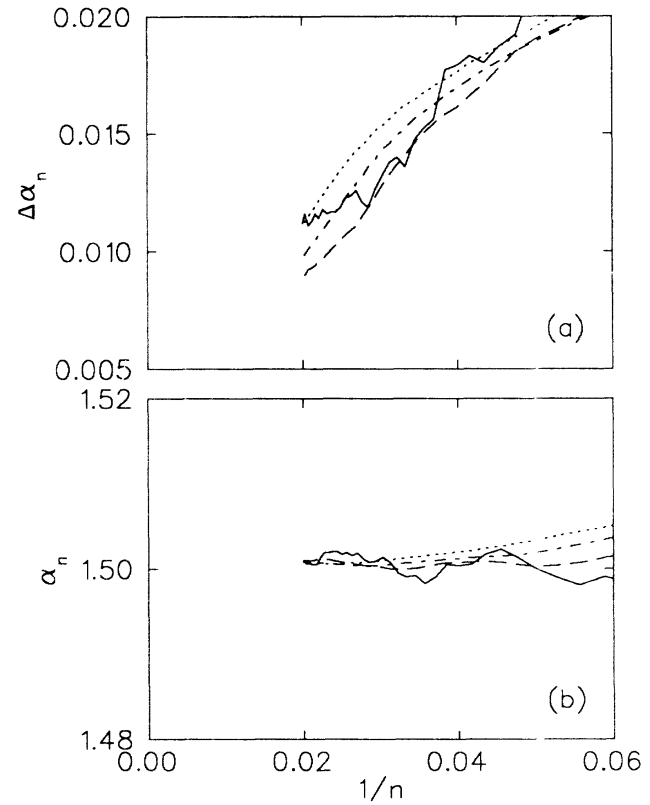

FIG. 1. Standard deviation (a) and average (b) of the estimates of the ratio method for series $A$ with $\sigma=0.05$ constant noise after smoothing transformation. (— $s=$ $2 ;--., s=3 ; \ldots,-, s=4$; and $\ldots, s=5$.) 
TABLE IV. Same as in Table III but for the method of differential approximants.

\begin{tabular}{|c|c|c|c|c|c|c|}
\hline \multirow[b]{2}{*}{ Function } & \multirow[b]{2}{*}{$\sigma$} & \multirow[b]{2}{*}{$N$} & \multicolumn{2}{|c|}{ Constant noise } & \multicolumn{2}{|c|}{ Linear noise } \\
\hline & & & $\alpha$ & $\Delta \alpha$ & $\alpha$ & $\Delta \alpha$ \\
\hline \multirow[t]{6}{*}{ A } & 0.01 & 20 & 1.501 & 0.069 & 1.529 & 0.102 \\
\hline & 0.05 & 20 & 1.566 & 0.181 & 1.598 & 0.225 \\
\hline & 0.20 & 20 & 1.507 & 0.423 & 1.557 & 0.336 \\
\hline & 0.01 & 50 & 1.503 & 0.067 & 1.497 & 0.049 \\
\hline & 0.05 & 50 & 1.503 & 0.143 & 1.508 & 0.154 \\
\hline & 0.20 & 50 & 1.506 & 0.670 & 1.557 & 0.642 \\
\hline \multirow[t]{6}{*}{$\mathrm{C}$} & 0.01 & 20 & 1.547 & 0.118 & 1.550 & 0.115 \\
\hline & 0.05 & 20 & 1.473 & 0.202 & 1.615 & 0.480 \\
\hline & 0.20 & 20 & 1.320 & 0.344 & 1.337 & 0.507 \\
\hline & 0.01 & 50 & 1.535 & 0.105 & 1.508 & 0.083 \\
\hline & 0.05 & 50 & 1.539 & 0.229 & 1.544 & 0.189 \\
\hline & 0.20 & 50 & 1.397 & 0.360 & 1.716 & 0.514 \\
\hline \multirow[t]{6}{*}{$\mathrm{G}$} & 0.01 & 20 & 1.388 & 0.065 & 1.407 & 0.060 \\
\hline & 0.05 & 20 & 1.474 & 0.159 & 1.484 & 0.156 \\
\hline & 0.20 & 20 & 1.492 & 0.370 & 1.564 & 0.397 \\
\hline & 0.01 & 50 & 1.508 & 0.046 & 1.517 & 0.052 \\
\hline & 0.05 & 50 & 1.535 & 0.200 & 1.572 & 0.253 \\
\hline & 0.20 & 50 & 1.532 & 0.617 & 1.582 & 0.523 \\
\hline \multirow[t]{6}{*}{$\mathrm{K}$} & 0.01 & 20 & 1.515 & 0.061 & 1.530 & 0.095 \\
\hline & 0.05 & 20 & 1.553 & 0.231 & 1.570 & 0.275 \\
\hline & 0.20 & 20 & 1.499 & 0.544 & 1.611 & 0.636 \\
\hline & 0.01 & 50 & 1.495 & 0.056 & 1.499 & 0.058 \\
\hline & 0.05 & 50 & 1.526 & 0.194 & 1.519 & 0.154 \\
\hline & 0.20 & 50 & 1.536 & 0.716 & 1.599 & 0.495 \\
\hline
\end{tabular}

TABLE V. Standard deviation of the estimates obtained by the Dlog-Padé and the differential approximant methods after a smoothing transformation, Eq. (2.7), on the test series with constant noise $(\sigma=0.05)$.

\begin{tabular}{cllllr}
\hline \hline \multirow{2}{*}{ Function } & \multicolumn{2}{c}{ Padé method } & \multicolumn{2}{c}{ DA method } \\
\hline $\mathrm{A}$ & $s$ & $N=20$ & $N=50$ & $N=20$ & $N=50$ \\
& 0 & 0.095 & 0.046 & 0.181 & 0.143 \\
& 1 & 0.113 & 0.063 & 0.165 & 0.087 \\
& 3 & 0.113 & 0.051 & 0.124 & 0.058 \\
$\mathrm{C}$ & 5 & 0.107 & 0.056 & 0.128 & 0.061 \\
& 0 & 0.247 & 0.061 & 0.202 & 0.229 \\
& 1 & 0.230 & 0.070 & 0.243 & 0.100 \\
& 3 & 0.226 & 0.066 & 0.215 & 0.069 \\
$\mathrm{G}$ & 5 & 0.239 & 0.073 & 0.161 & 0.079 \\
& & 0.093 & 0.069 & 0.159 & 0.200 \\
& 1 & 0.128 & 0.059 & 0.162 & 0.090 \\
& 3 & 0.131 & 0.063 & 0.122 & 0.059 \\
$\mathrm{~K}$ & 5 & 0.131 & 0.060 & 0.197 & 0.074 \\
& & & & & 0.194 \\
& 0 & 0.128 & 0.073 & 0.231 & 0.096 \\
& 1 & 0.135 & 0.075 & 0.273 & 0.062 \\
& 3 & 0.168 & 0.067 & 0.133 & 0.083 \\
\hline \hline
\end{tabular}




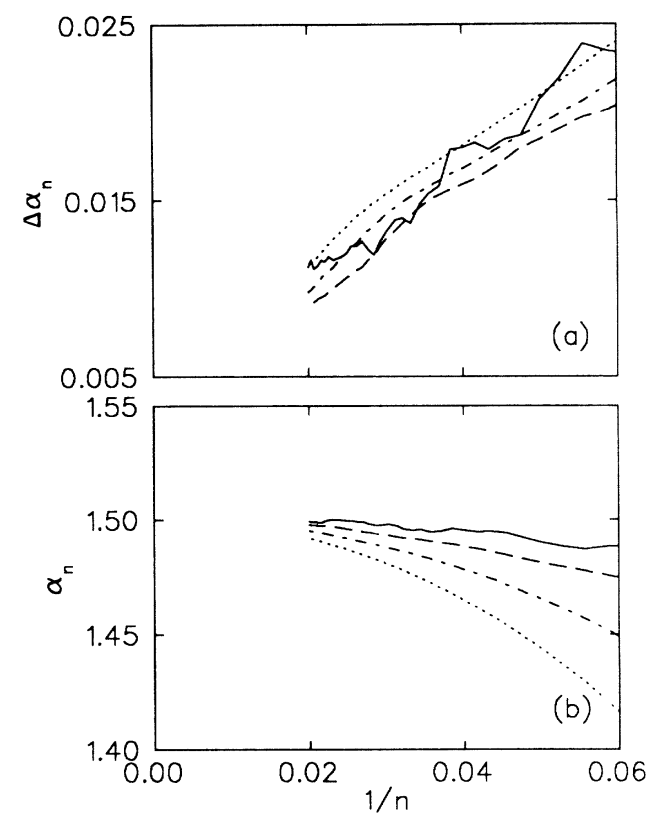

FIG. 2. Same as in Fig. 1 but for series $G$.

and $\alpha=1.52 \pm 0.05$ for series $C$ and $K$, respectively, where the uncertainties are mainly due to confluent singularities. For the function $K$ we repeated the investigation with linear noise, Eq. (3.3). As one can see from Fig. 6 the accuracy of the estimate is about the same as for constant noise, which is (also in this case) due to the confluent singularity, since the standard deviation [Fig. $6(\mathrm{a})$ - caused by stochasticity - is even smaller than before.

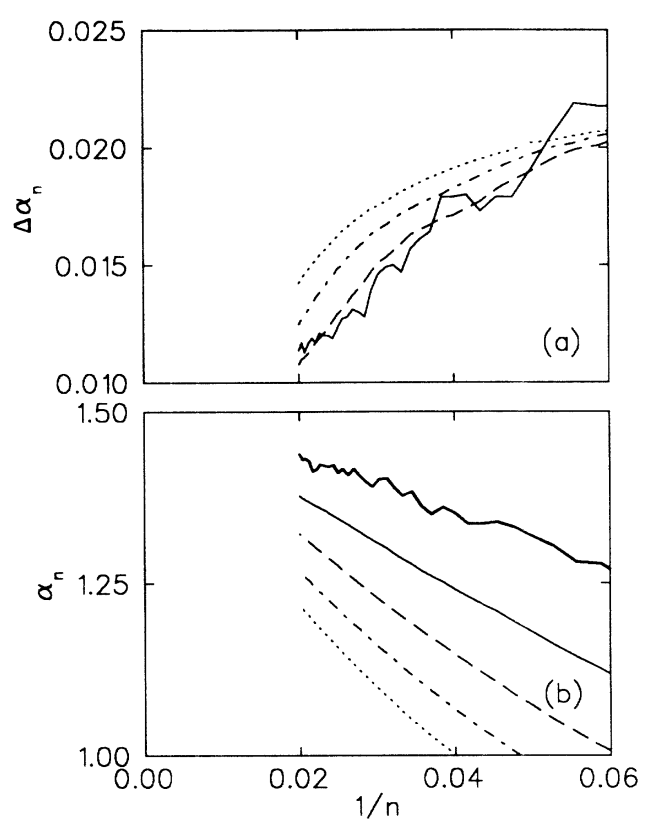

FIG. 4. Series $C$ with $\sigma=0.05$. Results for $s=1$ are denoted by thick line.

Concluding this part we mention another smoothing procedure based on a transformation that raises the series to the $p$ th power. This transformation, however, turned out to be far less effective than Eq. (2.7).

\section{Self-avoiding random walk}

As a final example we perform the analysis of the MC series of $R_{n}^{2}$ for self-avoiding random walks on the square lattice. In the simulation we generated 100 groups

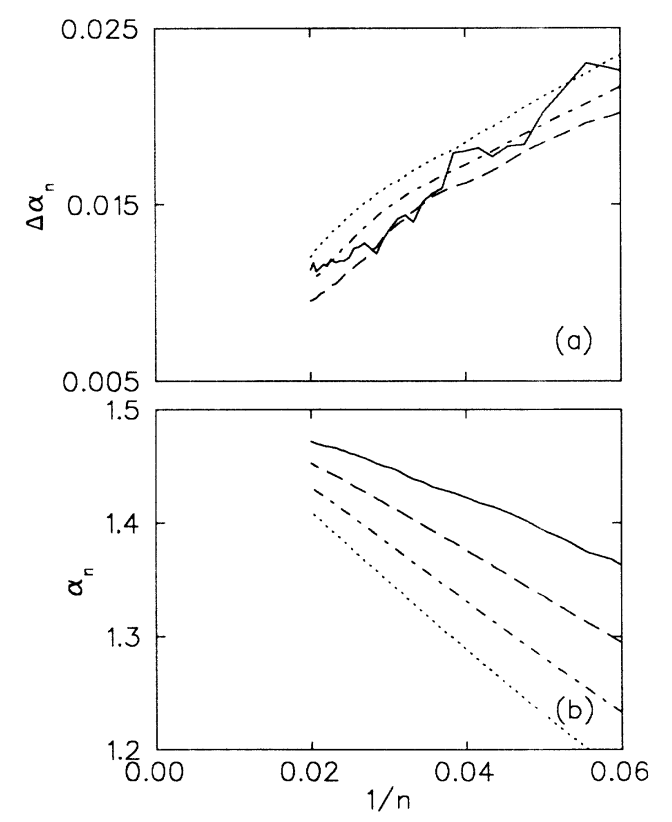

FIG. 5. Series $K$ with $\sigma=0.05$.
FIG. 3. Series $G$ with $\sigma=0.2$.

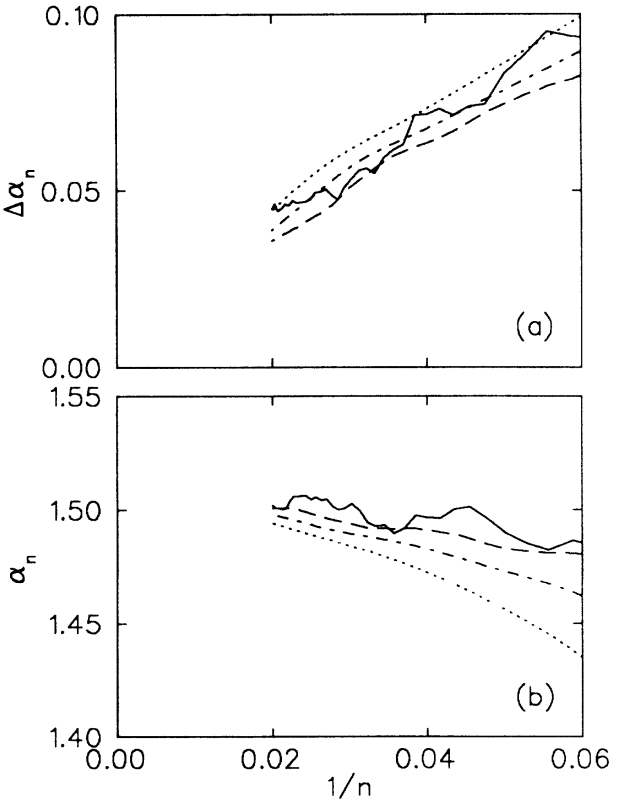


TABLE VI. Critical exponent of the end-to-end distance of the self-avoiding random walk on the square lattice obtained by analysing MC series of 10000 and 100000 started walks, respectively, after a smoothing transformation.

\begin{tabular}{llllll}
\hline \hline \multirow{2}{*}{ Walks } & \multicolumn{2}{c}{ Padé method } & \multicolumn{2}{c}{ DA method } \\
\hline 10000 & $s$ & $2 \nu+s$ & $\Delta(2 \nu)$ & $2 \nu+s$ & $\Delta(2 \nu)$ \\
& 0 & 1.494 & 0.237 & 1.604 & 0.410 \\
& 1 & 2.522 & 0.232 & 2.590 & 0.362 \\
& 3 & 4.500 & 0.233 & 4.471 & 0.389 \\
100000 & 5 & 6.508 & 0.225 & 6.563 & 0.474 \\
& 0 & 1.505 & 0.070 & 1.514 & 0.153 \\
& 1 & 2.502 & 0.072 & 2.526 & 0.116 \\
& 3 & 4.504 & 0.068 & 4.512 & 0.173 \\
& 5 & 6.504 & 0.065 & 6.522 & 0.192 \\
\hline \hline
\end{tabular}

of 100000 starting walks for $\mathrm{n}=6,10,14, \ldots, 50$, and performed the same analysis as before. To check the effect of noise we repeated the procedure with only 10000 starting walks per group. ${ }^{13}$ The results obtained by the Padé and DA methods are given in Table VI, while the estimates of the ratio method are drawn in Fig. 7.

For this problem the Pade method seems to give the most accurate estimate $2 \nu=1.5 \pm 0.07$, close to the exact one, $2 \nu=1.5 .^{14}$ The uncertainty of the DA method is about twice as large, and in this case the smoothing does not help too much. The estimates of the ratio method with smoothing (Fig. 7) show a strong systematic $1 / n$ dependence, which signals the presence of a strong confluent singularity. Due to this effect the uncertainty $( \pm 0.1)$ is not smaller than that of the Padé method.

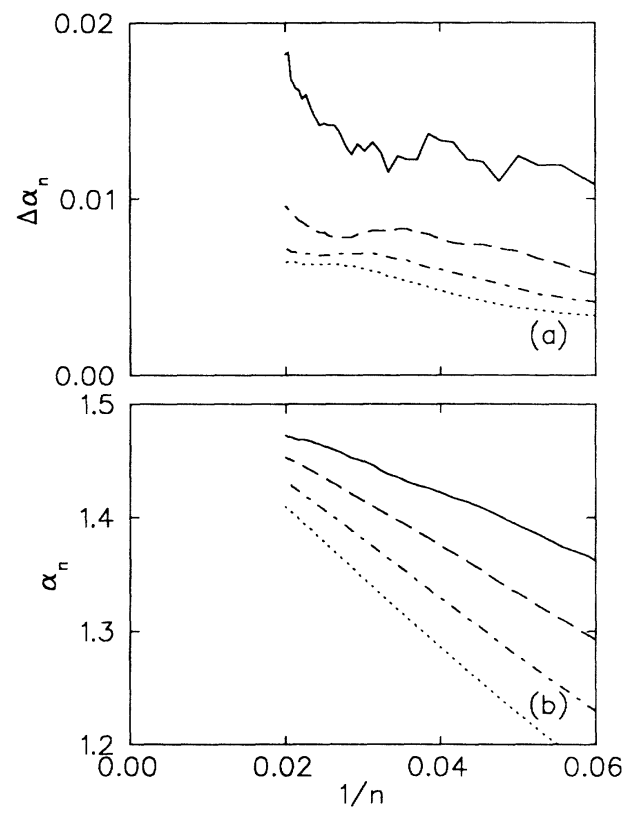

FIG. 6. Series $K$ with linear noise $(\sigma=0.05)$.

\section{DISCUSSION}

In this paper different methods have been applied to analyze power series of singular quantities generated by $\mathrm{MC}$ simulation. There are two sources of disturbances which make the analyses difficult for these series: confluent singularities and fluctuations in the individual coefficients. The most accurate methods developed for exact series reduce optimally the effect of confluent singularities, but-as we have observed on the example of differential approximants--one can only achieve a moderate success with these methods for series with noise.

On the other hand, the noise, the second source of problems, can be successfully reduced by different smoothing transformations. We found the ratio method,
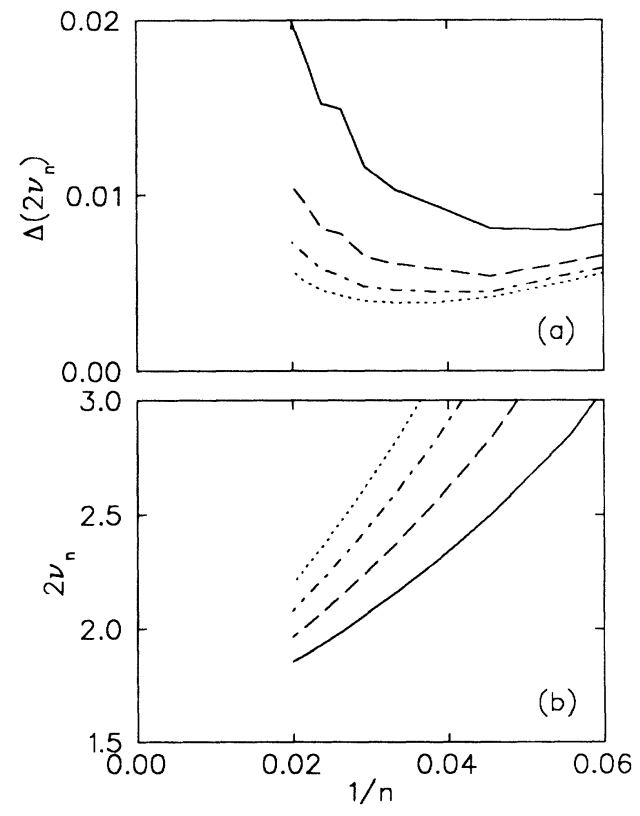

FIG. 7. Estimate and standard deviation for $2 \nu$ from a MC simulation of 100000 started self-avoiding random walks on the square lattice by the ratio method, using various values of smoothing. 
combined with optimal smoothing, to give the most accurate estimates for series, where the effect of confluent singularity is small. This method, however, loses its efficiency if the confluent singularity is strong, since the smoothing transformation makes its effect even stronger. For these cases the Padé method seems to be superior as we have seen in the example of the self-avoiding random walk. As a conclusion MC series have to be studied by different methods, at the same time including smoothing transformation as well, to clarify the roles played by the confluent singularity and the noise. The most reliable estimates are generally obtained by the ratio method with smoothing and-if the confluent singularity is strong-by the Padé method.

\section{ACKNOWLEDGMENTS}

This work was supported by the Sonderforschungsbereich 341 Köln-Aachen-Jülich. F.I. is grateful to J. Zittartz for hospitality in Köln.
*Permanent address: Central Research Institute for Physics, H-1525 Budapest, Hungary.

${ }^{1}$ For a review see Phase Transitions and Critical Phenomena, edited by C. Domb and M.S. Green (Academic, New York, 1974), Vol. 3.

${ }^{2}$ D.S. Gaunt and A.J. Guttmann, in Ref. 1; A.J. Guttmann, in Phase Transitions and Critical Phenomena, edited by C. Domb and J.L. Lebowitz (Academic, New York, 1989), Vol. 13.

${ }^{3}$ D.L. Hunter and G.A. Baker, Jr., Phys. Rev. B 7, 3346 (1973).

${ }^{4}$ R. Dekeyser, A. Maritan, and A.L. Stella, Phys. Rev. B 31, 4659 (1985); A 36, 2338 (1987).

${ }^{5}$ D. Stauffer, Introduction to Percolation Theory (Taylor and Francis, London, 1985).

${ }^{6}$ P.M. Lam, Phys. Rev. A 34, 2339 (1986).

${ }^{7}$ For an extensive review on Monte Carlo simulations, see Monte Carlo Methods, edited by K. Binder (SpringerVerlag, Berlin, 1979).

${ }^{8}$ F. Iglói, J. Phys. A 19, 3077 (1986); for a generalization outside of the critical point, see F. Seno and A.L. Stella, J. Phys. (Paris) 49, 739 (1988).

${ }^{9}$ M.N. Barber, in Phase Transitions and Critical Phenomena, edited by C. Domb and J.L. Lebowitz (Academic, New York, 1983), Vol. 8.

${ }^{10}$ D.L. Hunter and G.A. Baker, Jr., Phys. Rev. B 19, 3808 (1979); M.E. Fisher and H. Au-Yang, J. Phys. A 12, 1677 (1979).

${ }^{11}$ P.G. De Gennes, Scaling Concepts in Polymer Physics (Cornell University Press, Ithaca, 1979).

${ }^{12}$ This observation is in accordance with Eqs. (2.9) and (2.10); furthermore, for additive confluent singularity, the correction increases linearly with $s$.

${ }^{13}$ In the algorithm we fixed the number of starting walks and took into account only those which were self-avoiding after $n$ steps. As a consequence the uncertainty of the coefficients increases with $n$; we found an asymptotic behavior $\Delta \sigma(n) \sim n^{3}$.

${ }^{14}$ B. Nienhuis, Phys. Rev. Lett. 49, 1062 (1982). 